\title{
Gestión Energética en el Sector Salud en Colombia: Un Caso de Desarrollo Limpio y Sostenible
}

\author{
David Martinez-Sierra( ${ }^{(1)}$, Martha García-Samper ${ }^{(2) \star}$, Hugo Hernández-Palma ${ }^{(3)}$ y Willian Niebles-Nuñez ${ }^{(4)}$ \\ (1) Universidad Simón Bolívar, Facultad de Ingeniería, Barranquilla-Colombia. \\ (e-mail: dmartinez@unisimonbolivar.edu.co) \\ (2) Universidad de la Costa, Departamento de Gestión Organizacional, Barranquilla-Colombia. \\ (e-mail: mgarcia20@cuc.edu.co) \\ (3) Universidad del Atlántico, Programa Administración de Empresas, Barranquilla-Colombia. \\ (e-mail: hugohernandezpalma@gmail.com) \\ (4) Universidad de Sucre, Programa de Administración de Empresas, Sincelejo-Colombia. \\ (e-mail: williamniebles@yahoo.com.mx)
}

* Autor a quien debe ser dirigida la correspondencia.

Recibido Ene. 21, 2019; Aceptado Mar. 8, 2019; Versión final Abr. 8, 2019, Publicado Oct. 2019

\begin{abstract}
Resumen
El objetivo del presente artículo es analizar la gestión energética desde la mirada del desarrollo limpio y sostenible en las instituciones prestadoras de servicios de salud en Colombia. El estudio es realizado a través de un enfoque cualitativo con un método de revisión documental de la literatura con información de artículos científicos de los últimos 8 años, ubicados en bases de datos especializadas, esto con el propósito de explorar avances en esta materia a nivel mundial y determinar los aportes que pudiesen ser replicados en el país. Los resultados evidencian la existencia de un respaldo institucional para el desarrollo de la gestión energética, así como, la definición de diversas alternativas y áreas de intervención en función de los diferentes servicios prestados. Se concluye que las fuentes de mayor consumo energético están concentradas en climatización e iluminación, y que las estrategias para reducir costos se relacionan con la optimización de contratos de suministro de energía e instalaciones de centros hospitalarios.
\end{abstract}

\section{Energy Management in the Health Sector of Colombia: A Case of Clean and Sustainable Development}

\begin{abstract}
The objective of this article is to analyze energy management from the perspective of clean and sustainable development in institutions that provide health services in Colombia. The study is carried out through a qualitative approach with a method of documentary review of the literature with information from scientific articles of the last 8 years, located in specialized databases, this with the purpose of exploring advances in this area worldwide and determine the contributions that could be replicated in the country. Results show that there is evidence of institutional support for the development of energy management, as well as the definition of various alternatives and areas of intervention depending on the different services provided. It is concluded that the sources of greatest energy consumption are concentrated in air conditioning and lighting, and that strategies to reduce costs are related to the optimization of energy supply contracts and hospital facilities.
\end{abstract}

Keywords: energy management; health sector; innovation; sustainability; clean development 


\section{INTRODUCCIÓN}

Desde que los métodos para la producción y distribución de energía se sofisticaron, estos han contribuido notablemente al desarrollo de la humanidad, facilitando así que los diferentes sectores productivos que conforman la sociedad moderna tuviesen un acceso a fuentes de poder fiable y estable, permitiéndoles de este modo llevar a cabo sus actividades operativas sin contratiempos. Sin embargo, la tecnología empleada para la generación de este elemento ha estado impactando considerablemente el medio ambiente durante las últimas décadas. Adicionalmente, los cambios en el patrón de consumo de los seres humanos, ha venido aumentando considerablemente la urgencia por crear procesos más eficientes y amables ecológicamente (Cámpora, 2015). Por esta razón, el propósito central del estudio es abordar desde una perspectiva teórica el rol de las instituciones del sector salud en Colombia de cara a los grandes retos que se presentan en lograr una gestión energética adecuada.

En este contexto, diversas organizaciones a nivel internacional han puesto la mirada en los problemas ambientales y la creación de soluciones tangibles en el corto, mediano y largo plazo que ayuden a mitigar el impacto que tiene la actividad humana sobre todas las formas de vida que se encuentran en la naturaleza. Por ello, los acuerdos suscritos por diferentes Estados persiguen garantizar una mejora en la calidad de vida del hombre, con el uso de métodos de producción responsables que protejan los recursos no renovables y la estabilidad de los ecosistemas; En este sentido, el componente energético como parte de este sistema, desempeña un papel fundamental en la construcción de nuevas alternativas para la sostenibilidad futura. A su vez, la tecnología sin lugar a dudas ha contribuido a este propósito mediante el diseño de dispositivos que logran capitalizar y transformar las fuerzas de la naturaleza, tales como la energía fotovoltaica, el viento, el agua e incluso el aprovechamiento de residuos sólidos (Montiel y Bohórquez, 2019) en energía limpia y apta para su utilización en toda la gama y complejidad que representa la actividad humana en el mundo (Kalkan et al., 2012). No obstante, a pesar de estos avances en materia política y tecnológica, aún se requiere unir la voluntad de todos los sectores sociales para obtener resultados de mayor contundencia ante los problemas que se desarrollan actualmente en el campo energético.

Recientemente, fue expedido el estudio Regulatory Indicators for Sustainable Energy (RISE), en donde se evalúan indicadores de políticas de energía sostenible en diferentes países alrededor del mundo. Éste se constituye en el primer estudio relacionado con este tema y el cual evalúa aspectos como el acceso a la energía, la eficiencia energética y energía de fuentes renovables (Abdallah et al., 2013). Esta referencia es un esfuerzo por encaminar a los seres humanos hacia prácticas de consumo energético de mayor sostenibilidad, eficiente y amigable con el ambiente, con base en una serie de políticas universales en función de lograr los objetivos climáticos globales y el sétimo Objetivo de Desarrollo Sostenible (ODS) sobre la energía limpia (Programa de las Naciones Unidas para el Desarrollo, 2012). En este contexto, hasta el año 2017 alrededor de 50 países han definido y articulado marcos normativos que permiten la incorporación de la energía renovable como una alternativa eficaz; en comparación con el año 2010 donde apenas la mitad de estos estaban dispuestos a suscribir políticas en este sentido (Banco Mundial, 2018). Los datos del informe sugieren que se han reportado avances en esta materia pues en el 2017 el $93 \%$ de los países participantes en el estudio RISE se comprometieron a cumplir con metas oficiales para adoptar la energía renovable, contrastando con las cifras del año 2010 en donde solo se inclinaba un 37\% de estos. Según datos del RISE, específicamente para el año 2017, el 44\% de 137 países a nivel global contaban con un desempeño pobre en términos de la eficiencia energética, destacando que Somalia, Mozanbique y Liberia como las peores naciones en este indicador (Banco mundial, 2018); por su parte, Colombia se encuentra en el segmento del $32 \%$ de países que cuentan con marcos normativos intermedios de eficiencia en este período.

Todos los componentes citados se consideran de gran importancia por el momento actual, donde el tratamiento y atención del medio ambiente se vuelven esenciales, permeando las políticas nacionales. Según la oficina del Secretario General de las Naciones Unidas, al planeta le urge lograr la transición a las energías más limpias, proceso que permitirá suministrar servicios más eficientes a todos, crear empleo, garantizar la atención de salud y la educación, y posibilitar el crecimiento de las economías; un elemento clave para esa transformación es el aumento del uso de fuentes renovables (Calderón y Aguirre, 2017). La dramática situación ambiental que se experimenta en el presente siglo obliga a realizar esfuerzos mancomunados en todos los niveles y sectores de la sociedad para lograr mitigar así el impacto potencial que tendría en las generaciones futuras el uso inadecuado de los recursos naturales.

\section{CONTEXTO ENERGÉTICO DEL SECTOR EN COLOMBIA}

De acuerdo al informe RISE, en el contexto Latinoamericano los países mejor evaluados en una escala de 100 puntos para el año 2017 son Chile (77), Brasil (74) y Ecuador (61), por su parte, Colombia un puntaje de 60 , donde apenas el $24 \%$ proviene de energías renovables, lo que indica que es necesario llevar a cabo mayores esfuerzos por incorporar tecnología adecuada que permita emplear generación limpia que no afecte 
el medio ambiente. En contraparte, Venezuela (48) y Paraguay (55), son países de la región que se encuentran con un desempeño menor a Colombia, donde Venezuela alcanza apenas un $13 \%$ de energía renovable, mientras que Paraguay, a pesar de generar mayor cantidad de este tipo de alternativa para el consumo eléctrico (62\%), presenta carencias significativas en áreas como redes y transmisión, gestión de riesgos, planeación y expansión, entre otros aspectos.

Bajo este contexto, el sector salud es uno de los más sensibles en temas de consumo energético y por tanto puede observar un gran beneficio en la aplicación medidas que conduzcan a la optimización del empleo de combustibles de tipo fósil, es decir el rédito que generaría una inversión de este tipo puede llegar a ser bastante representativo en el área financiera. Investigaciones señalan el impacto medioambiental que tienen las operaciones de los centros de asistenciales en temas de salud pública (Burstein, 2016), en este sentido, sería congruente que estas organizaciones se esfuercen por conducir acciones en beneficio del interés común ecológico. Las tendencias en el desarrollo de alternativas para la producción y utilización de energía proveniente de fuentes más limpias y eficientes están llegando al sector salud. En Colombia los denominados hospitales verdes, caracterizados por incorporar políticas y estrategias para reducir el consumo de combustibles fósiles y hacer de estas estructuras un ejemplo de sostenibilidad, están señalando el camino para construir nuevos escenarios representando una iniciativa para mejorar la gestión de la salud ambiental en hospitales de la red pública, incorporando elementos de salud y seguridad para el medio ambiente, trabajadores, pacientes y la comunidad en la que ejerce su zona de influencia (Cuellar y Rodríguez, 2017). En este sentido, la renovación de los procesos por medio de asesorías técnicas en pro de la procura de prácticas que incluyan la tecnología limpia y segura es parte de los objetivos que se persigue actualmente el Estado colombiano a través de los organismos oficiales pertinentes.

El sector salud es consciente del impacto que producen sus acciones en el medio ambiente, además de los riesgos biológicos inherentes a su actividad, está claramente preocupado por reducir las emisiones de carbono y el consumo de energía de origen fósil, dado que este tipo de edificaciones son unas de las que más demanda energética en la categoría residencial (Papadopoulos, 2016). Para alcanzar este propósito, la energía, el consumo hídrico y la gestión ambiental conforman los ejes fundamentales en los cuales se adelantan acciones para obtener soluciones a mediano y corto plazo (Burstein, 2016); por otra parte, la construcción y el diseño de las estructuras haciendo uso de las más avanzadas técnicas de arquitectura y urbanismos, está permitiendo que las edificaciones cuenten con mayor potencialidad para aprovechar los recursos naturales. En virtud de lo anterior, existen áreas de oportunidad en las cuales las instituciones de salud pueden explorar para disminuir su huella ambiental, entre estas, se podría mencionar el control automático de la apertura de puertas y ventanas en aquellos espacios del recinto que cuenten con distintas temperaturas, esto con la finalidad de no sobre exigir a los equipos de aire acondicionado, reduciendo así el consumo energético; la revisión continua de los equipos dedicados a la incineración de material médico quirúrgico o biológico, controlando las emisiones y desechos potencialmente peligrosos; incorporación de modificaciones que empleen luz artificial con bajo consumo en los lugares donde sea viable; análisis del gasto energético de los equipos electrónicos para verificar su cumplimiento de acuerdo a los estándares internacionales, entre muchas otras medidas (Bakar, 2015). Estas acciones requieren entonces la asignación de todo tipo recursos y el compromiso de la dirección de la institución, acompañada de un cambio en la cultura de consumo de todos los usuarios, por ello, la concientización se convierte en otro de los aspectos clave de mejora para el éxito de este tipo de iniciativas (Saidel et al., 2006).

Todas estas posibles medidas para optimizar el consumo de energía en el sector salud están en sintonía con el desarrollo de ciudades modernas, las cuales persiguen dar respuesta a las necesidades de sus habitantes por medio de la optimización de los recursos. Por tal razón, existe una articulación importante entre todos los actores y sectores sociales, partiendo de lo establecido en los ODS hasta las políticas de orden nacional y local (Banco Mundial 2018). Al tomar acciones para que las instituciones de salud logren aprovechar las bondades de los recursos renovables, estas podrían impactar directa e indirectamente la población que atiende y en la comunidad, contribuyendo muy probablemente a la mejora de la calidad de vida de los ciudadanos. Por estas y otras razones, resulta relevante realizar un acercamiento a cómo el sector salud podría hacerse de los avances tecnológicos existentes en la actualidad para rediseñar sus patrones de consumo de energía. Este impacto en el contexto no solo viene dado por los beneficios en términos de ahorro energético, sino además en la creación de nuevas oportunidades para el desarrollo de la industria conexa, es decir, el sector tecnológico de apoyo para la construcción de las alternativas de generación de energía, tales como paneles fotovoltaicos, sistemas hídricos, diseño urbanístico y arquitectónico, entre otros (Abdallah, 2013). En este orden de ideas, cabe señalar que las mejoras no solo afectan la institución, sino también al entorno y desde variedad de arista, es decir, en las dimensiones social, económica y ambiental; por tanto, promover la concientización sobre el uso de energías renovables y sus beneficios, proveer de capacitación 
para implementar este tipo de transformaciones y crear políticas gubernamentales que incentiven la eficiencia energética podrían representar sin duda alguna los mecanismos idóneos para el sector salud se apropie de estas oportunidades.

En particular, las instituciones prestadoras de salud (IPS), al ser entidades de constante flujo de público y pacientes requiere un sostenimiento permanente y óptimo de las condiciones ambientales de sus instalaciones, así mismo es fundamental que la conservación ideal de los equipos utilizados para la prestación de servicios de consultas, urgencias, quirófanos, oficinas, laboratorios, entre otros (Madroñal et al., 2016). En los últimos años se han venido desarrollando tendencias que estimulan al sector a transformar sus políticas medioambientales para estar en sintonía con las directrices a nivel internacional y nacional que se están promulgando. La energía limpia ya no es una temática de moda, sino que se ha convertido en una necesidad imperiosa ante el peligro latente en el que se encuentra el planeta por las acciones del ser humano. Obviar esta realidad, representaría a largo plazo un alto impacto, no solo para el hombre, sino también para todas las especies con las que cohabita, provocando daños difíciles de reparar y obligando a comprometer mayor cantidad de recursos lo que podría afectar el equilibrio de la vida. Todo lo anterior respalda la importancia que tiene el análisis de la gestión energética en el sector salud desde el punto de vista del desarrollo limpio y sostenible, dado que forma parte integral del sistema económico y social, el cual no es ajeno a las políticas y acciones que se están impulsando en esta área. Para exponer tal relevancia, el artículo se apoya en una revisión exhaustiva de la literatura que permitirá contrastar las posiciones teóricas y los más recientes hallazgos sobre el uso eficiente de la energía en las organizaciones sanitarias y todas las potencialidades que podrían ser explotadas.

\section{METODOLOGÍA}

Para el presente artículo se estableció un enfoque cualitativo, que permitiera analizar el objeto de interés de una perspectiva global y con mayor énfasis en el contenido. En virtud de ello, el estudio aborda la gestión energética desde una perspectiva teórica que ayude a comprender sus componentes aplicables al sector salud en Colombia. Por ende, se detallan los resultados tomando en consideración artículos científicos que orientan sobre los avances que se ha logrado en esta materia en los últimos años, identificando además los puntos clave que puedan servir de apoyo para las instituciones sanitarias. En este orden ideas, el razonamiento inductivo sirvió de base para indagar en las reflexiones de los diferentes autores explorados y así llegar a establecer las conclusiones del caso. Por lo tanto, se definió una metodología de revisión documental, que se basó en la consulta de información generada los últimos 8 años en el área de investigación relacionada con la energía, consumo y ahorro energético en el sector salud a nivel global con miras a tomar los avances más significativos que pudiesen ser replicables al contexto de las IPS colombianas. Como fuente de consulta se utilizaron artículos científicos de bases de datos universitarias y científicas, tales como Springer, Scielo, Emerald, Scopus, entre otras; ubicando información relacionada con las palabras clave gestión energética, eficiencia, sector salud, sostenibilidad, energía limpia, y consumo energético, tanto a nivel local, regional, nacional e internacional. Para ello, se emplearon como criterios de inclusión artículos en revistas de corriente principal en idioma inglés y español de publicación reciente entre 2013 y 2018. Localizándose alrededor de unas 50 publicaciones y analizando los de mayor relevancia; una vez capturada la información, se procedió a clasificar la misma en orden de relevancia de acuerdo al objetivo planteado para la realización del documento final.

\section{RESULTADOS}

En Colombia al consultar la situación sobre la utilización de energía eléctrica, es común encontrar en primera instancia que existe una normativa vigente que se observa como marco regulatorio en todos los sectores productivos. Es así como se puede hacer mención de la Ley 1715 de 2014; la cual fue expedida con el fin de buscar el desarrollo de tres objetivos fundamentales y el establecimiento de estímulos económicos que motiven la inversión en la producción y/o utilización de energía a partir de fuentes no convencionales (Patarroyo, 2014): Promover el desarrollo y la utilización fuentes alternas; buscar la gestión óptima de la energía mediante la eficiencia y la respuesta de la demanda; y reducir emisiones de gases de efecto invernadero.

En particular para hoteles, clínicas y hospitales, Bancoldex creó en el 2015 una línea de crédito con el fin de promover proyectos de eficiencia energética validados por el Instituto Colombiano de Normas Técnicas y Certificación (ICONTEC) en estos sectores, esta financiación se encuentra orientada en inversiones para cambios de calderas, cambios de aires acondicionados, sistemas de control de aire acondicionado, cogeneración de energía, energía para el calentamiento de agua y climatización de piscinas con energía solar (Cuellar y Rodríguez, 2017). La gestión energética se constituye entonces en una herramienta para conseguir la máxima eficiencia en el suministro, conversión y utilización de los recursos naturales; ésta se conforma por una serie de fases (Gu et al., 2014) las cuales se muestran seguidamente en la figura 1. 


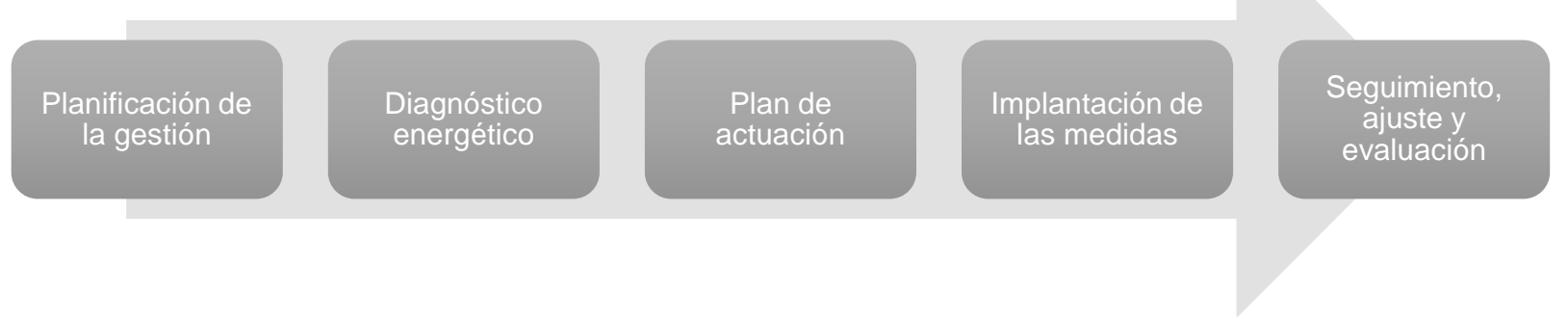

Fig. 1: Fases de la gestión energética, adaptada de Gu et al., 2018

Para poder determinar la eficiencia de un programa de ahorro energético, es necesaria la aplicación de indicadores de gestión. Éstos son herramientas que permiten medir los objetivos planteados (Bakar et al., 2015). El objeto es realizar la medición y contar con información confiable. En la tabla 1, se pueden observar un conjunto de medidas utilizadas actualmente a nivel mundial como una forma de evaluar los planes de ahorro y eficiencia en el uso de la energía.

Tabla 1: Indicadores de gestión energética

\begin{tabular}{|c|c|}
\hline Indicador & Descripción \\
\hline Consumo energético anual & $\begin{array}{l}\text { Permite medir la cantidad de energía que se usa en un año en una } \\
\text { edificación (Cárdenas et al., 2015). }\end{array}$ \\
\hline (\%) Ahorro combustible & $\begin{array}{l}\text { Se refiere al porcentaje de ahorro de combustible logrado con respecto } \\
\text { al año anterior (Cámpora, 2015). }\end{array}$ \\
\hline Gastos Energéticos(pesos) & Significa cuánto dinero se gasta por el consumo de energéticos. \\
\hline (\%) Ahorro respecto al año anterior & $\begin{array}{l}\text { Porcentaje de ahorro debido al uso de energía obtenido comparando } \\
\text { con el año anterior. }\end{array}$ \\
\hline $\begin{array}{l}\text { Consumo final de energía desagregado } \\
\text { por sectores }\end{array}$ & $\begin{array}{l}\text { El indicador permite conocer patrones de consumo de los diferentes } \\
\text { sectores y constituye además una importante herramienta para el } \\
\text { desarrollo de planes o programas que apunten al uso eficiente o ahorro } \\
\text { de energía (Correa et al., 2014). }\end{array}$ \\
\hline $\begin{array}{l}\text { Relación crecimiento del PIB y consumo } \\
\text { energético }\end{array}$ & $\begin{array}{l}\text { Relaciona el crecimiento económico con el consumo de energías fósiles } \\
\text { y las emisiones contaminantes generadas. Se dice que existe una } \\
\text { relación positiva, a mayor crecimiento económico, mayor consumo de } \\
\text { energías fósiles y mayor número de emisiones contaminantes (Al- } \\
\text { Mulali, et al., 2015). }\end{array}$ \\
\hline $\begin{array}{l}\text { Diagrama de dispersión del consumo } \\
\text { mensual de energía eléctrica vs camas- } \\
\text { día-ocupadas }\end{array}$ & $\begin{array}{l}\text { Evalúa si existe una dependencia directa entre el consumo energético } \\
\text { y las habitaciones/días/ocupación. Para que un índice de consumo sea } \\
\text { válido debe existir una correlación significativa entre el consumo de } \\
\text { energía y la variable, con la cual éste se relaciona. Para que un índice } \\
\text { sea válido como indicador de eficiencia energética el coeficiente de } \\
\text { correlación R2 entre las variables relacionadas en el índice debe ser } \\
\text { igual o mayor que } 0,75 \text { (González et al., 2017). }\end{array}$ \\
\hline Índices de gastos energéticos vs ingresos & $\begin{array}{l}\text { Indicador global integrador y útil, que no permite evaluar los resultados } \\
\text { específicos en cuanto a eficiencia en la utilización de los energéticos, ni } \\
\text { permite diagnosticar y corregir las causas que puedan provocar su } \\
\text { deterioro (Purroy et al., 2016). }\end{array}$ \\
\hline Consumo total de energía secundaria & $\begin{array}{l}\text { Mide la cantidad de energía secundaria que se consume en una } \\
\text { edificación en un año. }\end{array}$ \\
\hline Consumo de energía per cápita & $\begin{array}{l}\text { Mide el consumo aparente que surge del cociente entre consumo final } \\
\text { de energía (primaria y secundaria) de todos los sectores de la economía } \\
\text { y el número de habitantes para cada año (Liddle, 2014). }\end{array}$ \\
\hline $\begin{array}{l}\text { Emisiones de dióxido de carbono por } \\
\text { MWH generado }\end{array}$ & $\begin{array}{l}\text { Este indicador permite medir la intensidad de emisión de } \mathrm{CO} 2 \text {, uno de } \\
\text { los principales gases de efecto invernadero durante el proceso de } \\
\text { generación de energía eléctrica (Espinosa et al., 2017). }\end{array}$ \\
\hline
\end{tabular}


Tabla 1 (Continuación)

\begin{tabular}{|l|l|}
\hline $\begin{array}{l}\text { Índices de consumo por habitación-día- } \\
\text { ocupada (KWH/Hdo, M3/Hdo) }\end{array}$ & $\begin{array}{l}\text { Se busca correlacionar el consumo total de un edificio con respecto al } \\
\text { número de habitaciones ocupadas por día, ponderando aquellos factores } \\
\text { que marcan diferencias entre habitaciones del mismo edificio, por otras } \\
\text { cargas no asociadas con las habitaciones ocupadas, etc. (González et } \\
\text { al., 2017). }\end{array}$ \\
\hline $\begin{array}{l}\text { Índice de consumo por unidad de área } \\
\text { construida (KWH/m2-año). }\end{array}$ & $\begin{array}{l}\text { Busca correlacionar el consumo total de una edificación con respecto al } \\
\text { área construida de la misma (Allouhi et al., 2015). }\end{array}$ \\
\hline
\end{tabular}

El sector salud abarca una diversidad de instalaciones y su consumo energético depende del tipo de servicios que ofrecen a sus usuarios: consultas, urgencias, quirófanos, oficinas, laboratorios, entre otros (Burstein, 2016). Al respecto, Ahmed et al. (2014) establecieron que las instituciones de salud deben clasificarse según seis criterios principales: a) tipos de pacientes que se atiende; b) tipo de atención prestada; c) administración y propiedad; d) nivel de atención; e) tamaño de la estructura y f) ubicación geográfica de la edificación. De ahí que se hayan tipificado varias alternativas de intervención que contribuyen a la eficiencia energética en sus procesos, varias de las más destacables se ilustran seguidamente en la figura 2.

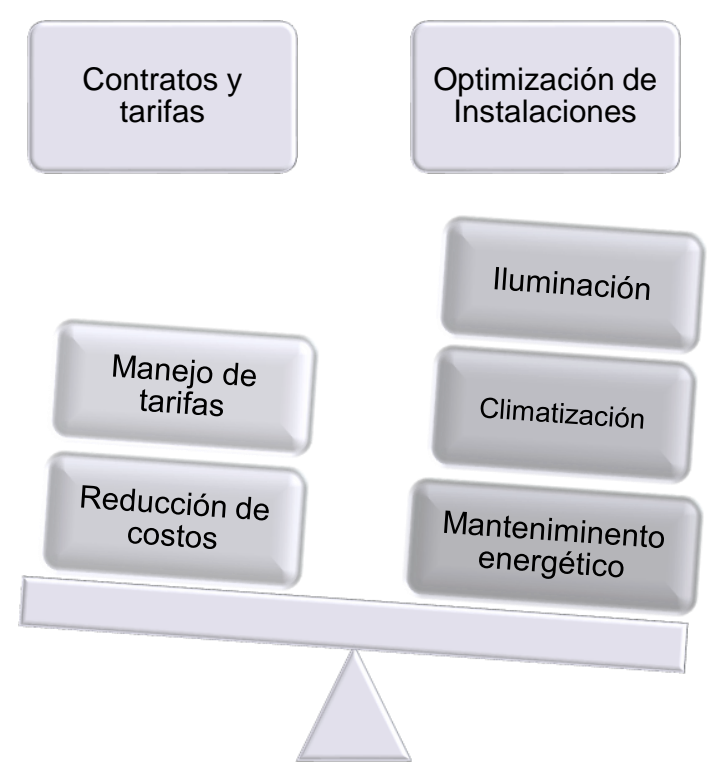

Fig. 2: Alternativas de intervención energética para el sector salud

Al hablar de optimizar el contrato se hace referencia al análisis y la descripción tarifaria con el fin de reducir los costos en términos de la presión, el grupo tarifario y/o el consumo (Sun y Nie, 2015). Por otro lado, la mejora de las instalaciones y su correspondiente tamaño óptimo, tiene que ver con las posibles intervenciones en aspectos físicos como la iluminación, climatización, aprovechamiento de calor que además deben ir en función de los servicios que presta la institución, a fin adecuar las necesidades de la población atendida con el consumo energético real requerido para garantizar la salud (García et al., 2014). En lo que respecta a la iluminación, las reducciones en el consumo eléctrico oscilan entre el $20 \%$ y el $85 \%$, asociado al uso de partes o elementos más eficientes, sistemas de control y aprovechamiento de la luz natural; además, se puede conseguir un ahorro adicional en el aire acondicionado, ya que la iluminación de bajo consumo energético presenta una menor emisión de calor (Chua, et al., 2013).

Son aplicables medidas como: uso de lámparas fluorescentes con balastos electrónicos (On/Off y Regulables) (Gómez, 2017; Teke y Timur, 2014); uso de lámparas de descarga; uso de lámparas fluorescentes compactas; sustituciones luminarias; uso de la luz natural; sistemas de control y regulación (control de horario, presencia y de luminosidad combinado) (Bambarén y Alatrista, 2016). Por su parte, por medio de la climatización se pueden conseguir ahorros entre un $10 \%$ y un $40 \%$ gracias a la optimización de las instalaciones, las características constructivas, ubicación, orientación del edificio, cerramientos, fachadas, cubiertas, carpintería, acristalamiento, protecciones solares, entre otras; implementación de un buen sistema de control y regulación; poner en práctica un modelo de free-cooling, de tal forma que se aproveche el intercambio de aire entre el exterior e interior con el fin de aprovechar una capacidad gratuita de refrigeración (Kalkan et al., 2015). 
De igual forma, se destacan otras medidas como el aprovechamiento de calor de los grupos de frío mediante el calentamiento que generan los condensadores de los frigoríficos para la producción de agua a elevadas temperaturas requeridas en otras áreas; instalación de recuperadores que permiten un intercambio de energía entre el aire extraído del edificio y el del exterior que se reintroduce para renovar el aire interior y el uso de bombas como un sistema reversible que puede suministrar calor o frío a partir de una fuente externa cuya temperatura es inferior o superior a la del local a calentar o refrigerar, utilizando para ello una cantidad de trabajo comparativamente pequeña (Montero et al., 2016).

Así también, se presenta como opción la optimización del rendimiento de las calderas a través de un buen dimensionamiento y la adecuación de su potencia a la demanda, mediante aquellas de baja temperatura y de condensación; estas últimas están diseñadas para recuperar más calor del combustible quemado que una convencional, y en particular, recuperar energía del vapor de agua que se produce durante la combustión (Abreu et al., 2016); Asimismo, en cuanto a la sustitución de gasóleo por gas natural se evidencia que, aunque el combustible utilizado principalmente en el sector es el gas natural, aún es posible encontrar calderas de gasóleo. Lo anterior permite afianzar cuatro factores cruciales que se muestran a continuación en la figura 3.

Otro componente clave es el agua caliente sanitaria (ACS): La producción de ACS se realiza generalmente mediante calderas, el ahorro de líquido no solo conlleva una disminución del insumo en sí mismo, sino también un decremento energético importante debido al poco consumo de combustible necesario para su calentamiento; además, el uso de reguladores eléctricos para el control de la velocidad de los motores que accionan las bombas eléctricas, consiguiendo ahorros hasta del $40 \%$ a 50\% (Pitarch et al., 2017). Asimismo, González et al. (2016) han señalado la importancia de reducir el consumo de agua en hospitales como una medida para reducir el impacto ambiental y aumentar la eficiencia de la gestión, en este sentido, expone que las instalaciones hospitalarias podrían reducir en $5.600 .000 \mathrm{~m} 3$ el uso de este bien y unos $2.912 \mathrm{Mkw}$, evitando así la emisión de 22.400 toneladas de CO2. En la misma línea se encuentra la gestión y mantenimiento energético; si se realizan acciones preventivas adecuadas, disminuirá la necesidad de llevar a cabo actividades correctivas constantes y, como resultado, se obtendrá un mejor rendimiento de la instalación, una reducción de costos y una mejor calidad de servicio (Guerrero et al., 2018);

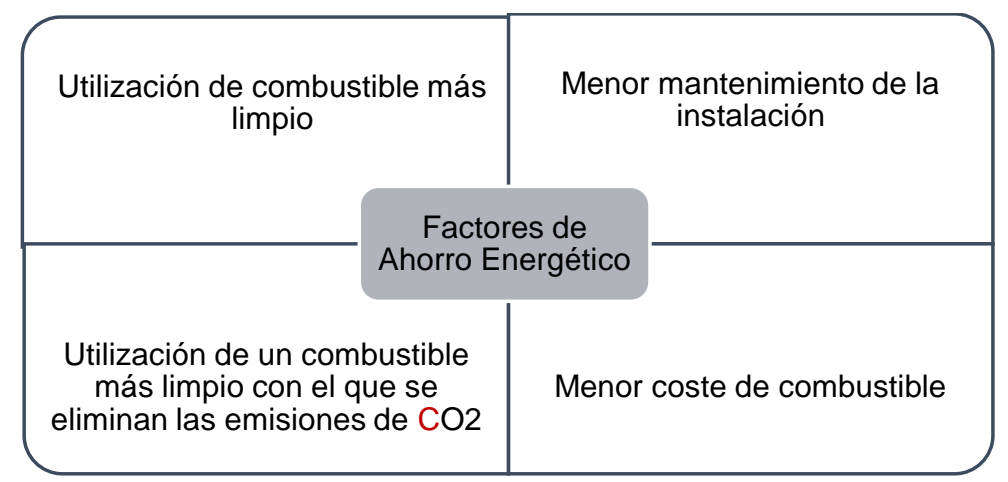

Fig. 3: Factores clave de ahorro energético en el sector salud, adaptada de Ferreira et al., 2015

Por otro lado, los sistemas de gestión, están basados en un ordenador y en un software, cuyo objetivo es obtener un uso más racional de las instalaciones, ahorrar energía, reducir mano de obra, evitar averías y prolongar la vida útil de los equipos como medidas principales. La Organización de las Naciones Unidas para el Desarrollo Industrial (ONUDI), ha recomendado a nivel mundial el uso de un sistema de gestión energética con base en el estándar internacional ISO 50001, cuyo cumplimiento se podrá realizar mediante la autoevaluación y autodeclaración de conformidad, y/o la certificación por entidad externa (Nordelo y Caminos, 2013). Algunas de las ventajas de su implementación son: a) Comprometer a la Alta Dirección y a la empresa con una política energética; b) Garantizar espacios de discusión, metodología para priorizar inversiones, recursos financieros, recursos humanos y tiempo para la discusión del tema energético; y c) Establecer y monitorear: indicadores de desempeño energético, una línea base, metas y objetivos reales de acuerdo a los criterios propios de la organización (Ricardo et al., 2015).

Adicional a todos estos aspectos, la concientización de la población en torno al uso adecuado de los servicios de salud, la ampliación de la red hospitalaria por parte del Estado y el cambio de paradigma en la atención de los pacientes por parte de los profesionales, son elementos clave para desarrollar una cultura energética integral que involucre a todos los grupos de interés (usuarios, personal de la salud e instituciones públicas) (Wang et al., 2016). En este sentido, el éxito de las alternativas de intervención energética (ver fig. 2) pasa por educar a los pacientes para que no asistan a hospitales de alta complejidad cuando no es necesario, sino que por el contrario utilicen las redes de atención primaria más cercanas a su localidad o empleando avances tecnológicos recientes como las redes inalámbricas de área corporal (Wireless Body Area Network [WBAN]), 
las cuales se utilizan para ayudar a reconocer problemas de salud de manera preventiva (Al-Janabi et al., 2016), construyendo así una conciencia y compromiso social orientada a la solución de los problemas energéticos (Parkhill et al., 2015). Por otra parte, con este tipo de iniciativas se estaría descongestionando la concentración de casos que se procesan en las estructuras hospitalarias y, por ende, se lograría administrar de forma más eficiente los recursos disponibles.

\section{CONCLUSIONES}

A partir de los avances citados y analizados, se generan las siguientes conclusiones: 1) las fuentes de mayor consumo energético en estas instalaciones de salud a nivel global están concentradas en los temas de climatización e iluminación; 2) algunas de las estrategias que pueden ser contempladas en las IPS colombianas para obtener una reducción en los costos de consumo de energía van relacionados con la mejora de contratos de suministro de energía, y la optimización de las instalaciones del centro hospitalario; 3) en las IPS de Colombia surgen alternativas de intervención en iluminación, climatización, agua caliente sanitaría, mantenimiento óptimo de instalaciones y equipos, implantación y uso eficiente de energía renovables, entre otros; 4) en este tipo de instituciones de salud, se reconoce la importancia de identificar los factores de mayor consumo energético y la forma en la cual pueden ser intervenidos para obtener una eficiencia, no solo en lo relacionado al gasto de este rubro, sino también en el costo económico que esto conlleva.

\section{REFERENCIAS}

Abdallah, K. B., M. Belloumi y D. De Wolf, Indicators for Sustainable Energy Development: A Multivariate Cointegration and Causality Analysis from Tunisian Road Transport Sector, https://doi.org/10.1016/j.rser.2013.03.066, Renewable and Sustainable Energy Reviews, 25, 34-43 (2013)

Abreu, H., M. González y otros tres autores, Evaluación de Esquemas de Cogeneración de Energía a partir de Bagazo de Caña de Azúcar, Centro Azúcar, 43(1), 87-98 (2016)

Ahmed, T.M., P. Rajagopalan y R. Fuller, A Classification of Healthcare Facilities: Toward the Development of Energy Performance Benchmarks for Day Surgery Centers in Australia. https://journals.sagepub.com/doi/abs/10.1177/1937586715575910, HERD: Health Environments Research \& Design Journal, 8(4), 139-157 (2015)

Al-Janabi, S., Al-Shourbaji y otros dos autores, Survey of Main Challenges (security and privacy) in Wireless Body Area Networks for Healthcare Applications, Egyptian Informatics Journal, 18(2), 113-122 (2017)

Allouhi, A., Y. El Fouih y otros cuatro autores, Energy Consumption and Efficiency in Buildings: Current Status and Future Trends, doi.org/10.1016/j.jclepro.2015.05.139, Journal of Cleaner production, 109, 118-130 (2015)

Al-Mulali, U., H.G. Fereidouni y otros dos autores, Examining the Bi-directional Long Run Relationship between Renewable Energy Consumption and GDP Growth, Renewable and Sustainable Energy Reviews, 22, 209-222 (2013)

Bakar, N.A., Hassan y otros cinco autores, Energy Efficiency Index as an Indicator for Measuring Building Energy Performance: A review, Renewable and Sustainable Energy Reviews, 44, 1-11 (2015)

Bambarén, C. y M.D. Alatrista, Huella de Carbono en Cinco Establecimientos de Salud del Tercer Nivel de Atención de Perú, 2013, Revista Peruana de Medicina Experimental y Salud Publica, 33(2), 274-277 (2016)

Banco Mundial, Regulatory Indicators for Sustainable Energy (RISE), The World Bank (2018)

Burstein, T., Rol del Sector Salud Ante el Cambio Climático, Revista Peruana de Medicina Experimental y Salud Publica, 33(1), 139-142 (2016)

Calderón, C.L. y J. Aguirre, Solar Cells as a Pedagogical Alternative in the Teaching of Electricity, Momento, (55), 44-56 (2017)

Cámpora, C., Rey Sol: La Alta Dependencia de los Combustibles Fósiles con Impacto Negativo Sobre el Ambiente Lleva a Pensar en la Integración de las Energías Renovables, como la Solar, para Disponer de Alternativas que Mejoren la Sostenibilidad de los Sistemas Ambientales, Productivos y Sociales, RIA, Revista de investigaciones agropecuarias, 41(1), 14-20 (2015)

Cárdenas, J. P., E. Muñoz y otros dos autores, Simplified Life Cycle Assessment Applied to Structural Insulated Panels Homes, Revista Ingeniería de Construcción, 30(1), 33-38 (2015)

CESMAG, I. U. y G. Nariño, Situación Actual del Uso Racional y Eficiente de la Energía en Colombia, Libros Editorial, UNIMAR (2015)

Cuellar, W. A. y S. R. Rodríguez, Colombia: Territorio de Inversión en Fuentes no Convencionales de Energía Renovable para la Generación Eléctrica, Ingeniería Investigación y Desarrollo: I2+ D, 17(1), 37-48 (2017)

Espinosa, S., D. A. Cazco y M. Y. Salcedo, Economic Dispatch Hydrothermal System with CO2 Emissions Constraints, IEEE Latin America Transactions, 15(11), 2090-2096 (2017)

Ferreira, W., L. Campos y otros dos autores, Environmental and Economic Impact of the Use of Natural Gas for Generating Electricity in the Amazon: A Case Study, Dyna, 82(190), 89-95 (2015) 
García-Sanz-Calcedo, J., F. López-Rodríguez y F. Cuadros, Quantitative Analysis on Energy Efficiency of Health Centers According to Their Size, Energy and Buildings, 73, 7-12 (2014)

Gómez C., Modelo de Negocio para una Solución Inteligente en Optimización del Consumo Energético en Equipos VAC en Edificaciones del Sector Terciario, Tesis de Maestría, Universidad de la Costa, Departamento de Gestión Industrial, Agroindustrial y Operaciones (2017)

González, A.G., J. García-Sanz-Calcedo y otros dos autores, A Quantitative Analysis of Cold Water for Human Consumption in Hospitals in Spain, dx.doi.org/10.1155/2016/6534823, Journal of healthcare engineering (2016)

González, A.M., A. B. Nordelo y otros tres autores, Nuevos Índices de Consumo Energético para Hoteles Tropicales, Ingeniería Energética, 38(3), 198-207 (2017)

Gu, W., Z. Wu y otros cinco autores, Modeling, Planning and Optimal Energy Management of Combined Cooling, Heating and Power Microgrid: A Review, doi.org/10.1016/j.ijepes.2013.06.028, International Journal of Electrical Power \& Energy Systems, 54, 26-37 (2014)

Guerrero, J. W. G., A. D. Toscano y otros dos autores, Analysis of the Energetic and Productive Effects Derived by the Installation of a Conveyor Belt in the Metal-mechanic Industry, International Journal of Energy Economics and Policy, 8(6), 196-201 (2018)

Kalkan, N., E. A. Young y A. Celiktas, Solar Thermal Air Conditioning Technology Reducing the Footprint of Solar Thermal Air Conditioning, Renewable and Sustainable Energy Reviews, 16(8), 6352-6383 (2012)

Liddle, B., Impact of Population, Age Structure, and Urbanization on Carbon Emissions/Energy Consumption: Evidence from Macro-level, Cross-country Analyses, doi.org/10.1007/s11111-013-0198-4, Population and Environment, 35(3), 286$30(2014)$

Madroñal, M., B. J. Galeano y N. J. Escobar, Search for Facility Management's Kpis to Manage the Hospitals Infrastructure in Colombia, Revista Ingeniería Biomédica, 10(20), 13-19 (2016)

Montero, R., A. A. Legrá y J. R. Hechavarría, Optimización Operacional de Redes Hidráulicas para Climatización Centralizada de Hoteles, Ingeniería Hidráulica y Ambiental, 37(2), 3-17 (2016)

Montiel-Bohórquez, N.D. y J.F. Pérez, Generación de Energía a partir de Residuos Sólidos Urbanos. Estrategias Termodinámicas para Optimizar el Desempeño de Centrales Térmicas, Información Tecnológica, 30(1), 273-284 (2019)

Nordelo, A. B. y C. Caminos, Recomendaciones Metodológicas para la Implementación de Sistemas de Gestión de la Energía según la Norma ISO 50001, Editorial Universo Sur ISBN: 978-959 (2013)

Papadopoulos, A. M, Energy Efficiency in Hospitals: Historical Development, Trends and Perspectives, In Energy Performance of Buildings, 217-233, Springer, Cham, (2016)

Parkhill, K. A., F. Shirani y otros cuatro autores, We Are a Community [but] That Takes a Certain Amount of Energy': Exploring Shared Visions, Social Action, and Resilience in Place-based Community-led Energy Initiatives, Environmental science \& policy, 53, 60-69 (2015)

Patarroyo, M. R., Fomento de las Energías Renovables en Colombia: Entre Contradicciones e Iniciativas Fallidas, Revista Vasca de Administración Pública, Herri-Arduralaritzako Euskal Aldizkaria, (99), 2581-2604 (2014)

Pitarch, M., E. Navarro-Peris y otros dos autores, Experimental Study of a Subcritical Heat Pump Booster for Sanitary Hot Water Production Using a Subcooler in Order to Enhance the Efficiency of the System with a Natural Refrigerant (R290), doi.org/10.1016/j.jirefrig.2016.08.017, International Journal of Refrigeration, 73, 226-234 (2017)

Programa de las Naciones Unidas para el Desarrollo [UNPD], Objetivos de Desarrollo Sostenible, UNPD (2012)

Purroy, R., F. Gallardo y otros cuatro autores, Eficiencia Energética y Económica, Bienestar Familiar y Productividad en Agroecosistemas Tropicales, Agricultura, Sociedad y Desarrollo, 13(4) (2016)

Ricardo, H., A. Medina y Q. Núñez, Tendencias Internacionales y Nacionales en los Sistemas de Gestión Empresariales, Revista Universidad y Sociedad, 7(3), 40-46 (2015)

Saidel, M.A., L.N. Rossi y otros dos autores, Sistema para la Gestión del Uso de la Energía en Instituciones Públicas, Información Tecnológica, 17(4), 71-76 (2006)

Sun, P. y P. Y. Nie, A Comparative Study of Feed-in Tariff and Renewable Portfolio Standard Policy in Renewable Energy Industry, doi.org/10.1016/j.renene.2014.08.027, Renewable Energy, 74, 255-262 (2015)

Teke, A. y O. Timur, Assessing the Energy Efficiency Improvement Potentials of HVAC Systems Considering Economic and Environmental Aspects at the Hospitals, Renewable and Sustainable Energy Reviews, 33, 224-235 (2014)

Wang, T., X. Li y otros dos autores, Building Energy Efficiency for Public Hospitals and Healthcare Facilities in China: Barriers and Drivers, Energy, 103, 588-597 (2016) 
